\title{
Challenges of in vitro conservation of Citrus genetic resources
}

\author{
L.S. Samarina ${ }^{1 *}$, V.I. Malyarovskaya ${ }^{1}$, R.S. Rakhmangulov ${ }^{1}$, Y.L. Orlov ${ }^{2}$, \\ O.B. Dobrovolskaya ${ }^{2}$ \\ ${ }^{1}$ Russian Research Institute of Floriculture and Subtropical Crops, Sochi, Russia \\ ${ }^{2}$ Institute of Cytology and Genetics SB RAS, Novosibirsk, Russia \\ *e-mail: samarinalidia@gmail.com
}

Key words: Citrus, contamination, micropropagation, culture media, true-to-type, endophytes

Motivation and Aim: To preserve in vitro collections of plant genetic recourses including elite cultivars of tree crops, it is necessary to use vegetative buds and meristems as explants that are genetically identical to the stock plant. However, the stage of aseptic culture initiation of woody vegetative buds is associated with such challenges as a high contamination rate in vitro, a slow growth, and the appearance of secondary-infected explants. The main challenges in creating in vitro backup collection of Citrus cultivars are a high degree of fungal contamination of vegetative buds and the subsequent decrease in the growth potential of plantlets in vitro. It is well known, woody plants, in particular Citrus species, are in close cohabitation with fungal microorganisms. Surface sterilization of explants does not relieve tissue from internal infection, on the cultural medium the hyphae of the fungus leave the plant tissues and proliferate on culture media, which inhibits the development of explants in vitro [1]. In this regard, the aim of our work is to study the effectiveness of various tools of decontamination of vegetative Citrus explants for initiation in vitro culture and development of a reliable in vitro conservation.

Methods and algorithms: Pre-cultivation techniques, pre-treatment of cuttings with fungicides, gradual sterilization, addition of antibiotics to the nutrient medium, as well as micro-grafting were tested for establish efficient tissue culture initiation of elite lemon cultivars of collection FGBSI RRIFSC (Sochi, Russia). The studies were carried out on Citrus limon (L.) Burm cultivars. Axillary buds with shoot segment of $0.5-0.7 \mathrm{~cm}$ long were taken as explants.

Results: The highest rate of sterile explants $32-42 \%$, was obtained by pre-cultivation the cuttings in a incubating chamber at $22{ }^{\circ} \mathrm{C}$, followed by pretreatment with fungicides, using gradual sterilization and adding tetracycline $400 \mathrm{mg} / 1$ to the culture medium. However, after the third passage of subculture and conservation, the viability of the plantlets was reduced, leaves dropped and plantlets died within 6 months of in vitro conservation. Using in vitro micrografting technique, it was possible to overcome the problem of viability losses of bud explants, but this method is extremely labor- and timeconsuming, only 1 of 300 manipulations was successful.

Conclusion: Thus, reliable preservation of valuable citrus genotypes in collection in vitro is currently problematic due to close cohabitation with fungal microorganisms and low efficiency of micropropagation as a whole.

\section{References}

1. Samarina L.S. (2013) Optimization of micropropagation and conservation protocols for lemon cultivars in vitro. PhD Thesis / Russian State Agrarian University. Moscow. 